\title{
TWO THEOREMS ON HYPERHYPERSIMPLE SETS
}

\author{
BY \\ ROBERT W. ROBINSON( ${ }^{(1)}$
}

There are two main results. First, there is a hyperhypersimple set which is not quasimaximal. Second, there is an $r$-maximal set which is not hyperhypersimple. These results answer questions raised by Young [10, pp. 75 and 81] and McLaughlin [3, p. 87]. McLaughlin [4] reports a weaker, "non-co-r.e." version of our second result, due to D. A. Martin. He also improves Martin's result, though the "co-r.e." versions of both would be equivalent to our second result. Lachlan [2] has obtained different proofs of the two main results, as discussed in the last section.

The proof of the first result involves a construction closely akin to the maximal set construction as handled by Yates [8]. The priority system applicable to maximal set constructions was introduced by Friedberg [1], and is essentially unaltered. The proof of the second result requires that the maximal set priorities be altered in an essential way, and thus represents more of a departure.

1. Preliminaries. We are concerned only with recursively enumerable (r.e.) subsets of the nonnegative integers. Definitions of the special classes of r.e. sets which we deal with may be found in [3], [5], and [6]. By an observation of Yates $[8$, p. 344] it is clear that any quasimaximal set is hyperhypersimple. The examples of hyperhypersimple sets appearing in the literature, as in [8], are all quasimaximal. Thus our first construction provides a new variety of hyperhypersimple set. The only examples of $r$-maximal sets (r.e. sets maximal with respect to the recursive sets) previously known are just maximal sets. Every maximal set is certainly $r$-maximal. The converse of this triviality is disposed of by our second construction.

The set of all nonnegative integers is denoted by $N$, and the empty set by $\varnothing$. All of the sets under consideration will be subsets of $N$, all functions of one variable will be subsets of $N \times N$, etc. All quantification is to be taken over $N$ or some indicated subset of $N$. A relation is called recursive (in the exhibited variables) if its representing function is recursive (in the corresponding variables), and a set or sequence of sets is called recursive if its membership relation is recursive. A sequence of sets $S(i)$ is called r.e. just if there is some recursive sequence $S(i, s)$ such that $S(i)=\bigcup S(i, s)(s \geqq 0)$ for all $i$.

Presented to the Society, October 30, 1965 under the title Two recursively enumerable sets; received by the editors February 1, 1966 and, in revised form, September 30, 1966.

(1) The material in this paper appears in the author's doctoral dissertation at Cornell University. The author is indebted to his thesis advisor, Gerald E. Sacks, for many useful conversations, and to the referee for helpful suggestions. This work was done while the author was a National Science Foundation fellow. 
Let $W(i, s)$ be a recursive sequence such that if $W(i)=\bigcup W(i, s)(s \geqq 0)$ for all $i$, then $\{W(i) \mid i \geqq 0\}$ is the set of all r.e. sets. It is further required that

(1) $(y)(i)(s)(y \in W(i, s) \Rightarrow y \leqq s)$ and

(2) $(i)(s)(W(i, s) \subseteq W(i, s+1))$.

Such a sequence is obtained by taking $W(i, s)$ to be the set of integers placed in the $i$ th r.e. set by the sth stage of some standard enumeration of the r.e. sets.

Also we require a specific pairing function $p$, defined by $p(x, y)=\frac{1}{2}(x+y)$ $\cdot(x+y+1)+x$, along with the inverses $r$ and $c$. These satisfy the relation $p(r(x), c(x))=x$ for all $x$. The functions $p, r$, and $c$ are all recursive, and $p$ maps $N \times N$ isomorphically onto $N$. The following three facts are assumed without mention in our proofs. First, for every $x$ the set $r^{-1}(x)$ is infinite. Next, $p(x, y) \geqq x$ for all $x$ and $y$. Finally, $p(x, y)>p(0, x+y)$ for all $y$ and all $x>0$.

2. THEOREM 1. There is a hyperhypersimple set which is not quasimaximal.

A recursive sequence of sets $H(i, s)$ is defined, along with a recursive predicate $P(k, s, x)$ and a recursive function $x(k, s)$, all simultaneously by induction on $s$. For a fixed $s$ the definition of $P(k, s, x)$ and $x(k, s)$ proceeds by simultaneous induction on $k$ for all $x$, with the help of an auxiliary recursive function.

Let $E(k, s, y)=\sum 2^{k-j}(j \leqq k \& y \in W(j, s))$ for all $k, s$, and $y$. It may be noted that $E(k, s, y)$ is the $k$-state of $y$ at stage $s$ in Friedberg's terminology [1]. An immediate result of the definition is

$$
(j)(k)(x)(y)(s)((j \geqq k \& E(k, s, x)>E(k, s, y)) \Rightarrow E(j, s, x)>E(j, s, y)) .
$$

In view of (2) it also follows that

$$
(x)(k)(s)(t)(t \geqq s \Rightarrow E(k, t, x) \geqq E(k, s, x)) .
$$

At stage $s=0$, set $H(i, 0)=\varnothing$ for all $i$.

For any stage $s \geqq 0$, and any $k$ and $x$, we set

$$
\begin{gathered}
P(k, s, x) \equiv x \notin H(r(k), s) \&(j)(j<k \Rightarrow x(j, s)<x), \text { and } \\
x(k, s)=\mu x(P(k, s, x) \&(y)(P(k, s, y) \Rightarrow E(k, s, y) \leqq E(k, s, x))) .
\end{gathered}
$$

Finally, for any stage $s+1>0$, and any $i$, let

$$
H(i, s+1)=\{x \mid x \leqq s+1 \&(k)(x=x(k, s) \Rightarrow r(k)<i)\} .
$$

These definitions result in recursive sets, functions, and predicates, for in the definition of $x(k, s)$ the least number operator is always well defined, and the quantifier is implicitly bounded by $s$, due to (1). We conclude by setting

$$
H(i)=\bigcup H(i, s) \quad(s \geqq 0) .
$$

The construction may be viewed as a series of attempts at specifying the sets $\bar{H}(i)$. The approximation at stage $s$ to the set $\bar{H}(i)$ consists of the numbers $x(k, s)$ for 
all $k$ such that $r(k) \geqq i$. The choice of $x(k, s)$ is limited by the "availability predicate" $P$. This is because $P(k, s, x(k, s))$ must always be true, by (6). The first half of (5) insures that the sets $H(i)$ are nested, and the second half makes $H(i+1)-H(i)$ infinite for all $i$. Modulo the restriction imposed by $P$, the choice of $x(k, s)$ is determined by the function $E$. That is, $x(k, s)$ is chosen so as to maximize the value of $E(k, s, x(k, s))$. The function $E(k, s, x)$ is simply a convenient way of putting the highest priority on the membership of $x$ in $W(0)$, the next highest priority on membership in $W(1)$, and so on down to $W(k)$. This choice of priorities gives the r.e. sequence $H(0), H(1), \ldots$ properties strongly resembling maximality, as will be seen in Lemma 3.

Certain simple properties follow at once from the definitions. First, for any $s$ and $i, x(i, s)<x(i+1, s)$, so that the function $\lambda i x(i, s)$ is always $1-1$. Next, it is evident that for every $s$ and $i, H(i, s) \subseteq H(i+1, s)$, directly from the definition. Further, for every $s$ and $i, H(i, s) \subseteq H(i, s+1)$. For suppose $x \in H(i, s)$, and $x=x(k, s)$. Then $P(k, s, x)$ must be true, so that $x \notin H(r(k), s)$. Since $x \in H(i, s)$ and $H(i, s) \subseteq H(i+n, s)$ for all $n, i>r(k)$. If $x=x(j, s)$ then $j=k$, and so of course $i>r(j)$. Since $x \leqq s$ by virtue of $x \in H(i, s)$, it follows that $x \in H(i, s+1)$. From these two facts we see that $H(i) \subseteq H(i+1)$ for every $i$.

Lemma 1. For each $j, \lim _{s} x(j, s)$ exists.

Proof. We proceed by induction on $j$. Suppose, then, that $s_{0}$ is such that for all $s \geqq s_{0}$ and all $k<j$, we have $x(k, s)=x\left(k, s_{0}\right)$. The definition of $H(r(j), s+1)$ requires that $x(j, s) \notin H(r(j), s+1)$. By (6), $P(j, s, x(j, s))$ is true. If $s \geqq s_{0}$, the right half of (5) remains unchanged as it applies to the definition of $P(j, s+1, x(j, s))$, and so $P(j, s+1, x(j, s))$ is true. By (4) and (6) it follows that

$$
E(j, s+1, x(j, s+1)) \geqq E(j, s, x(j, s)),
$$

for all $s \geqq s_{0}$. As $\lambda s \lambda x E(j, s, x)$ can take on at most $2^{j+1}$ different values, there is a stage $s_{1} \geqq s_{0}$ such that if $s \geqq s_{1}$, then $E(j, s, x(j, s))=E\left(j, s_{1}, x\left(j, s_{1}\right)\right)$. But for all $s \geqq s_{1}$ it is now easy to see from (6) that $x(j, s+1) \leqq x(j, s)$, and so $\lim _{s} x(j, s)$ does exist.

Let $x(j)=\lim _{s} x(j, s)$ for all $j$. Since $x(0, s)<x(1, s)<\cdots$ for all $s$, then in the limit $x(0)<x(1)<\cdots$.

LEMMA 2. $N=\bigcup H(i)(i \geqq 0)$, and for all $i H(i+1)-H(i)=\{x(j) \mid r(j)=i\}$.

Proof. First, let $y$ be such that $y \notin\{x(j) \mid j \geqq 0\}$. Then we claim that $y \in H(0)$. By Lemma 1 we can find $s_{0}$ such that for any $s \geqq s_{0}$ and $k \leqq y, x(k, s+1)=x(k, s)$. It is readily seen that $y \leqq x(y, s)$ for every $s$, and of course $x(y, s)<x(y+1, s)<\cdots$. Thus for any $s \geqq s_{0}, y \notin\{x(j, s) \mid j \geqq 0\}$. So if $s_{1}=\max \left\{s_{0}, y\right\}$, it is clear that $y \in H\left(0, s_{1}\right)$.

Second, consider $y=x(j)$ for some $j$. As seen in the proof of Lemma $1, x(j, s)$ $\notin H(r(j), s+1)$ for all $s$. Thus in the limit $x(j) \notin H(r(j))$. By Lemma 1 , let $s_{0}$ be 
such that for all $s \geqq s_{0}, x(j, s)=x(j)$. Take $s_{1}=\max \left\{x(j), s_{0}\right\}$. Then it is clear that $x(j) \in H\left(r(j)+1, s_{1}\right)$, so that $x(j) \in H(r(j)+1)-H(r(j))$. Recalling that $H(k) \subseteq H(k+1)$ for all $k$, the lemma now follows easily.

LEMMA 3. For every e there is a number $i(e)$ such that for all $k, j \geqq i(e),(r(k)=r(j))$ $\Rightarrow(x(k) \in W(e) \Leftrightarrow x(j) \in W(e))$, and either (1) for all $i \geqq i(e), H(i+1)-H(i) \subseteq W(e)$, or (2) for all $i \geqq i(e),(H(i+1)-H(i)) \cap W(e)=\varnothing$.

Proof. Induct on $e$, supposing the lemma to hold for all $f<e$. Take $j_{0}=\max \{e\}$ $\cup\{i(f) \mid f<e\}$. Suppose the lemma to be false for $e$. Then there is a pair $j_{1}, j_{2}$ such that $j_{0}<j_{1}<j_{2}, r\left(j_{1}\right) \leqq r\left(j_{2}\right), x\left(j_{1}\right) \notin W(e)$, and $x\left(j_{2}\right) \in W(e)$. For if not, recalling that $r(j) \leqq j$ for all $j$, the construction of $i(e)$ would be routine. Let $s_{0}$ be such that if $s \geqq s_{0}, j \leqq j_{2}$, and $f \leqq e$, then $x(j, s)=x(j)$ and $x(j) \in W(f, s) \Leftrightarrow x(j) \in W(f)$. From the definitions of $j_{0}$ and $s_{0}$, we see that for any $s \geqq s_{0}$ and $f<e, E\left(f, s, x\left(j_{1}\right)\right)$ $=E\left(f, s, x\left(j_{2}\right)\right)$. Thus since $x\left(j_{1}\right) \notin W(e)$ and $x\left(j_{2}\right) \in W(e)$, we have that $E\left(e, s, x\left(j_{1}\right)\right)$ $<E\left(e, s, x\left(j_{2}\right)\right)$. By (3), $E\left(j_{1}, s, x\left(j_{1}\right)\right)<E\left(j_{1}, s, x\left(j_{2}\right)\right)$, or what is the same thing for $s \geqq s_{0}, E\left(j_{1}, s+1, x\left(j_{1}, s\right)\right)<E\left(j_{1}, s+1, x\left(j_{2}, s\right)\right)$. We have $H\left(r\left(j_{1}\right)\right) \subseteq H\left(r\left(j_{2}\right)\right)$ as $r\left(j_{1}\right) \leqq r\left(j_{2}\right)$, and since $j_{1}<j_{2}$ it follows that $P\left(j_{2}, s, y\right) \Rightarrow P\left(j_{1}, s, y\right)$ for any $y$. In particular $P\left(j_{2}, s+1, x\left(j_{2}, s+1\right)\right)$ must hold, and $x\left(j_{2}, s\right)=x\left(j_{2}, s+1\right)$ since $s \geqq s_{0}$, so that $P\left(j_{1}, s+1, x\left(j_{2}, s\right)\right)$ is true. But the latter along with $E\left(j_{1}, s+1, x\left(j_{1}, s\right)\right)$ $<E\left(j_{1}, s+1, x\left(j_{2}, s\right)\right)$ implies that $x\left(j_{1}, s\right) \neq x\left(j_{1}, s+1\right)$, where $s \geqq s_{0}$, a contradiction of the definition of $s_{0}$. Hence the lemma must hold for $e$.

We have already remarked that for all $i, H(i) \subseteq H(i+1)$. By Lemma 2 and the remark preceding it, $H(i+1)-H(i)$ is infinite for all $i$. Thus $H(i)$ cannot be quasimaximal for any $i$.

It remains to see that $H(0)$ is hyperhypersimple. First, we claim that $H(i+1)-H(i)$ is always cohesive. This is immediate from Lemma 2 and the first part of Lemma 3. Now, suppose that $H(0)$ were not hyperhypersimple. Then there would be an r.e. sequence $\{S(i) \mid i \geqq 0\}$ of pairwise disjoint r.e. sets such that $S(i) \cap \bar{H}(0)$ is infinite for all $i$. Let $K(e)=\{j \mid(H(j+1)-H(j)) \cap S(e)$ is infinite $\}$, for all $e$. We show that for any $e, K(e) \neq \varnothing$. Let $S(e)=W(k)$ and let $i(k)$ be as in Lemma 3. Then using Lemma 2 we see that $S(e) \subseteq \bar{H}(i(k))$ or else $S(e) \cap \bar{H}(i(k))=\varnothing$. In the former case $K(e)$ is infinite. In the latter case, $S(e) \subseteq H(i(k))$, and so $(H(i(k))-H(0)) \cap S(e)$ is infinite. Since $H(i(k))-H(0)$ is the finite disjoint union of $H(i(k))-H(i(k)-1), \ldots$, $H(1)-H(0)$, it follows that for some $j<i(k),(H(j+1)-H(j)) \cap S(e)$ is infinite. So $j \in K(e)$, and again $K(e) \neq \varnothing$. Now, if $i \neq j$ then $K(i) \cap K(j)=\varnothing$, because each set $H(e+1)-H(e)$ is cohesive, and $S(i) \cap S(j)=\varnothing$. Consider $S(E)=\bigcup S(2 i)(i \geqq 0)$, $S(Q)=\bigcup S(2 i+1) \quad(i \geqq 0), K(E)=\bigcup K(2 i)(i \geqq 0)$, and $K(Q)=\bigcup K(2 i+1)(i \geqq 0)$. $S(E)$ and $S(Q)$ are disjoint r.e. sets, and $K(E)$ and $K(Q)$ are infinite and disjoint. If $k \in K(E)$, then $(H(k+1)-H(k)) \cap S(E)$ is clearly infinite, and similarly for $Q$ in place of $E$. Suppose that $S(E)=W(e)$. Then Lemma 3 fails at $e$, for by our construction neither of the alternatives (1) or (2) can be true for any value of $i(e)$. By contradiction $H(0)$ must be hyperhypersimple, and so is $H(i)$ for all $i$. 
3. THEOREM 2. There is an r-maximal set which is not hyperhypersimple.

Sequences of sets $H(s)$ and $R(i, s)$, functions $x(j, s)$ and $E(j, s, y)$, and a predicate $P(j, s, x)$ are all defined simultaneously by induction on $s$. For each $s$, the definitions of $x(j, s), E(j, s, y)$, and $P(j, s, x)$ proceed by simultaneous induction on $j$, for all $x$ and $y$ at each step. The definition of $E(j, s, y)$ depends on whether or not $r(j)=0$.

At stage $s=0$ set $H(0)=\varnothing$ and $R(i, 0)=\varnothing$ for all $i \geqq 0$.

At any stage $s \geqq 0$, set

$$
E(p(0, d), s, y)=\sum 2^{d-k}(k \leqq d \& y \in W(k, s))
$$

for every $d$ and $y$. Next, for all $n$ and $i$ such that $n+i=d$ and $n>0$, set

$$
E(p(n, i), s, y)=\sum 2^{d-k}(k \leqq d \& y \in W(k, s) \& x(p(0, d), s) \in W(k, s)) .
$$

For all $x$ and $k$ let

(9) $P(k, s, x) \equiv(j)(j<k \Rightarrow x>x(j, s)) \&(x \notin R(0, s) \vee x \in R(r(k), s)-H(s))$, and for all $k$ let

$$
x(k, s)=\mu x(P(k, s, x) \&(y)(P(k, s, y) \Rightarrow E(k, s, x) \geqq E(k, s, y))) .
$$

Finally, at stage $s+1>0$, let

$$
\begin{aligned}
H(s+1) & =\{y \mid y \leqq s+1 \&(j)(y \neq x(j, s))\}, \\
R(0, s+1) & =\{y \mid y \leqq s+1 \&(j)(y=x(j, s) \Rightarrow r(j) \neq 0)\}, \quad \text { and } \\
R(i, s+1) & =\{y \mid y \leqq s+1 \&(j)(y=x(j, s) \Rightarrow r(j)=i)\}
\end{aligned}
$$

for all $i>0$.

The quantifiers employed in these definitions are implicitly bounded by $s$ or $k$, and the least number operator is easily seen to be well defined. Thus all our functions, sequences of sets, and predicates are recursive. In conclusion, we set $H=\bigcup H(s)(s \geqq 0)$ and $R(i)=\bigcup R(i, s)(s \geqq 0)$ for all $i$. These sets are then r.e., and of course the sequence $\{R(i) \mid i>0\}$ is r.e. also.

As in the construction of Theorem 1 , the predicate $P$ defined in (9) imposes certain restrictions on the choice of $x(k, s)$, since by (10) $P(k, s, x(k, s))$ must hold. Beyond this the choice of $x(k, s)$ is dictated by the priority scheme embodied in the definition of $E(k, s, x)$. If $r(k)=0$, (7) applies and the priority scheme is unchanged from Theorem 1. The numbers $x(k, s)$ for $r(k)=0$ constitute the sth approximation to $\bar{R}(0)$, and so $R(0)$ turns out to be maximal. But if $r(k)>0$, (8) applies and the highest priority goes to membership in $W(0, s)$ only if $x(p(0, n+j), s) \in W(0, s)$ (where $k=p(j, n))$. The same restriction may nullify the value of membership in $W(1), \ldots$, down to $W(n+j)$. This has the effect of rejecting, eventually, every set $W(k)$ such that $\bar{R}(0) \cap W(k)$ is finite. The numbers $x(k, s)$ for $r(k)=i>0$ represent the $s$ th approximation to the difference $R(i)-H$. The sets 
$R(i)-H$ are not cohesive; instead, the use of the restricted priority scheme yields that if $\bar{R}(0) \cap W(k)$ is infinite, then $\bar{H}-W(k)$ is finite, as seen in Lemma 5.

Directly from the definitions we see that $R(0, s)=\bigcup R(i, s)(i>0)$ for every $s$, and $H(s)=R(i, s) \cap R(j, s)$ for every $s, i$, and $j$ such that $i>0, j>0$, and $i \neq j$. Also for every $s, x(0, s)<x(1, s)<\cdots$. We claim that $R(i, s) \subseteq R(i, s+1)$ for every $s$ and every $i>0$. Suppose that $x \in R(i, s)$ with $i>0$. Then $x \leqq s$, and $P(k, s, x)$ is true only if $r(k)=i$. Consequently we cannot have $x=x(k, s)$ unless $r(k)=i$, and so by definition $x \in R(i, s+1)$. In addition, this yields that $H(s) \subseteq H(s+1)$ and $R(0, s)$ $\subseteq R(0, s+1)$ for all $s$. Going to the limit in $s$ we now see that $R(0)=\bigcup R(i)(i>0)$ and $H=R(i) \cap R(j)$ for all $0<i \neq j>0$.

LEMMA 4. $\operatorname{Lim}_{s} x(j, s)$ exists for all $j$.

Proof. Induct on $j$, assuming the lemma to hold for all $k<j$. Suppose, first of all, that $j=p(n, i)$ for some $i$ and some $n>0$. Then $p(n, i)>p(0, n+i)$. By the induction hypothesis there is a stage $s_{0}$ such that for all $s \geqq s_{0}$ and $k<j, x(k, s)$ $=x\left(k, s_{0}\right)$, and for all $e \leqq j, x(k, s) \in W(e, s) \Leftrightarrow x\left(k, s_{0}\right) \in W\left(e, s_{0}\right)$. In particular let $I=\{m \mid m \leqq n+i \& \quad . \quad(0, n+i) \in W(m)\}$. Then for $s \geqq s_{0}(8)$ becomes

$$
E(j, s, x)=\sum 2^{n+i-m}(\text { (n } \in I \& x \in W(m, s)),
$$

and hence we hate the analogue of (4) for our particular $j$ and $s_{0}$,

$$
(x)(t)(s)\left(t \geqq s \geqq s_{0} \Rightarrow E(j, t, x) \geqq E(j, s, x)\right) .
$$

It is easy to verify now, as in Lemma 1 , that for $s \geqq s_{0}, P(j, s+1, x(j, s))$ is always true. Thus we see that $E(j, s+1, x(j, s+1)) \geqq E(j, s, x(j, s))$. Of course for all $s$ and $x, E(j, s, x)<2^{j+1}$, and so $E(j, s, x(j, s))$ goes to a limit, say by stage $s_{1}>s_{0}$. Then for all $s \geqq s_{1}, x(j, s) \geqq x(j, s+1)$, and $\lim _{s} x(j, s)$ exists. Suppose, finally, that $j=p(0, d)$ for some $d$. Then (4) holds without alteration, and the proof proceeds as before.

Let $x(j)=\lim _{s} x(j, s)$ for all $j$. It is now easy to see that $\bar{R}(0)=\{x(p(0, n)) \mid n \geqq 0\}$, $R(i)-H=\{x(p(i, n)) \mid n \geqq 0\}$ for all $i>0$, and $\bar{H}=\{x(k) \mid k \geqq 0\}$. We omit the proof because of its similarity to that of Lemma 2 . These sets are all infinite since $x(0)<x(1)<\cdots$, which is the limit of a previously noted set of inequalities.

Further, $\bar{R}(0)$ is cohesive. We omit the proof of this because it would resemble the proof of Lemma 3 (as it bears on the first part of the lemma).

LEMMA 5. For any e such that $W(e) \cap \bar{R}(0)$ is infinite, there is a number $k(e)$ such that for all $k \geqq k(e), x(k) \in W(e)$.

Proof. For any $e$, let $d(e) \geqq e$ be such that either (1) for all $d \geqq d(e), x(p(0, d)) \in W(e)$ or (2) for all $d \geqq d(e), x(p(0, d)) \notin W(e)$. This is possible because $R(0)$ is maximal. Suppose the lemma to be true for all $f<e$. Let $h(e)$ be such that $p(0, h(e)) \geqq k(f)$ if $f<e$ and $W(f) \cap \bar{R}(0)$ is infinite, and $p(0, h(e)) \geqq p(0, d(f))$ for all $f \leqq e$. Then we 
claim that we can set $k(e)=p(0, h(e))$ if $W(e) \cap \bar{R}(0)$ is infinite. For in this case consider any $h \geqq p(0, h(e))$.

If $h=p(0, d)$ for some $d$, then $h \geqq p(0, h(e)) \geqq p(0, d(e))$, and $d \geqq d(e)$. Thus by definition of $d(e)$, since $W(e) \cap \bar{R}(0)$ is infinite, $x(h)=x(p(0, d)) \in W(e)$.

Suppose that for some $d, p(0, d)<h<p(0, d+1)$. Then $p(0, d) \geqq p(0, h(e))$, so that $d \geqq d(f)$ for all $f \leqq e$. Let $s_{0}$ be a stage such that for all $s \geqq s_{0}$, all $f \leqq e$, and all $j \leqq p(0, d+1)$ we have $x(j, s)=x(j)$ and $x(j) \in W(f, s) \Leftrightarrow x(j) \in W(f)$. So for all $f<e, x(p(0, d+1), s) \in W(f, s) \Leftrightarrow x(p(0, d), s) \in W(f, s)$. So if $x(h)=x(h, s) \notin W(e)$, then $E(h, s, x(h, s))<E(h, s, x(p(0, d+1), s))$, directly from (8) and the fact that $h \geqq p(0, d)$ where $d \geqq e$. But this is impossible, for $P(p(0, d+1), s, x(p(0, d+1), s))$ certainly holds, and hence $P(h, s, x(p(0, d+1), s))$, contradicting the definition of $x(h, s)$. Thus $x(h) \in W(e)$. Since we assumed only that $h \geqq p(0, h(e))$, the lemma is proved.

It is clear from Lemma 5 that $H$ is $r$-maximal. For, suppose $S$ is a recursive set. Then either $S \cap \bar{R}(0)$ or $\bar{S} \cap \bar{R}(0)$ is infinite; say, without loss of generality, that the latter is the case. Then $\bar{S}$ is r.e., so $\bar{S}=W(e)$ for some $e$. By Lemma $5, \bar{H}-W(e)$ is finite, and so $\bar{H} \cap S$ is finite.

Moreover, $H$ cannot be hyperhypersimple. For we can obviously construct an r.e. sequence $\{S(i) \mid i>0\}$ of pairwise disjoint r.e. sets such that for all $i>0$, $S(i) \subseteq R(i)$, and $\bigcup S(i)(i>0)=\bigcup R(i)(i>0)=R(0)$. Since $R(i) \cap R(j)=H$ for all $0<i \neq j>0$ and $R(i)-H$ is infinite for every $i>0$, this implies that $S(i) \cap \bar{H}$ is infinite for every $i>0$.

4. Related results. It has recently been shown by Lachlan [2, Theorem 2] that if $A$ and $B$ are r.e. sets and $A-B$ is hyperhyperimmune, then $A-B$ is co-r.e. In particular, the differences $H(i+1)-H(i)$ from the proof of Theorem 1 are comaximal, and so $H(0)$ is contained in each of infinitely many maximal sets, no two of which have a finite difference. In contrast to this, Lachlan [2, §3] constructs a hyperhypersimple set which is not contained in any maximal set. This provides an alternative proof of Theorem 1, since any quasimaximal set must be contained in a maximal set.

In addition, Lachlan [2, Theorem 7] shows that any maximal set contains a nonmaximal $r$-maximal set, and the theorem rephrased above shows that no such set is hyperhypersimple. In this indirect way Lachlan provides a stronger version of our Theorem 2.

\section{REFERENCES}

1. R. M. Friedberg, Three theorems on recursive enumeration, J. Symbolic Logic 23 (1958), 309-316.

2. A. H. Lachlan, On the lattice of recursively enumerable sets, Trans. Amer. Math. Soc., (to appear).

3. T. G. McLaughlin, Some observations on quasicohesive sets, Michigan Math. J. 11 (1964), 83-87. 
4. —_ Two remarks on indecomposable number sets, Z. Math. Logik Grundlagen Math. 12 (1966), 187-190.

5. E. L. Post, Recursively enumerable sets and their decision problems, Bull. Amer. Math. Soc. 50 (1944), 284-316.

6. G. F. Rose and J. S. Ullian, Approximation of functions on the integers, Pacific J. Math. 13 (1963), 693-701.

7. G. E. Sacks, A maximal set which is not complete, Michigan Math. J. 11 (1964), 193-205.

8. C. E. M. Yates, Recursively enumerable sets and retracing functions, Z. Math. Logik Grundlagen Math. 8 (1962), 331-345.

9. - Three theorems on the degrees of recursively enumerable sets, Duke Math. J. 32 (1965), 461-468.

10. P. R. Young, Linear orderings under one-one reducibility, J. Symbolic Logic 31 (1966), 70-85.

\section{Cornell University,} ITHACA, NEW YORK

INSTITUTE FOR ADVANCED STUDY, Princeton, New Jersey 